\title{
Ventricular Tachycardia during Treatment with Modafinil for Narcolepsy: A Case Report
}

\author{
Heleen Binnenmars ${ }^{1}$, Herman H. D. Idzerda ${ }^{1}$, Hanno L. Tan ${ }^{2}$, Gerard C. M. Linssen ${ }^{1 *}$ \\ ${ }^{1}$ Department of Cardiology, Hospital Group Twente, Almelo, The Netherlands; ${ }^{2}$ Department of Cardiology, Academic Medical Cen- \\ ter, Amsterdam, The Netherlands. \\ Email: *g.linssen@zgt.nl
}

Received August 10 ${ }^{\text {th }}, 2012$; revised September 24 $4^{\text {th }}$ 2012; accepted October $7^{\text {th }}, 2012$

\begin{abstract}
Pharmacological treatment of narcolepsy is complex. We reported a case of recurrent episodes of polymorphic ventricular tachycardia attributed to the use of modafinil, a recently approved wake-promoting agent for narcolepsy and shift work sleep disorder. Modafinil is also approved as adjunctive treatment of obstructive sleep apnea/hypopnea syndrome. While the exact mechanism of action for modafinil is not known, central dopamine receptors seem to play an essential role. Adverse influences on the electrocardiogram (ECG) or drug-related cardiac arrhythmias are rarely reported, but are considered as clinically important.
\end{abstract}

Keywords: Modafinil; Narcolepsy; Ventricular Tachycardia

\section{Introduction}

Narcolepsy, which affects 1 in 2000 people in the general population, is characterized by excessive daytime sleepiness (EDS), cataplexy, and other dissociated manifestations of rapid eye movement sleep [1,2]. Treating daytime sleepiness is complex, and determined by the precise nature of the sleep disorder. The disease is currently treated with amphetamine-like central nervous system stimulants (for EDS) and antidepressants (for cataplexy). The effects of modafinil are attributed to wake-promoting mechanisms via central dopamine receptors [3-6]. Adverse influences on the electrocardiogram (ECG) or cardiac arrhythmias are rarely reported [7]. We presented a case of modafinil-associated ventricular tachycardia.

\section{Case Report}

A 50-year-old man with known hypertension, chronic obstructive pulmonary disease, and narcolepsy presented to the emergency department with chest pain at rest and concomitant nausea. For several years, he had been taking irbesartan $150 \mathrm{mg}$, rabeprazol $20 \mathrm{mg}$, salmeterol/fluticason, salbutamol and, since a few weeks, modafinil 50 $\mathrm{mg}$. Until recently, he had used a daily dosage of $100 \mathrm{mg}$ of modafinil which he did not tolerate because of lightheadedness. He had never smoked, did not use alcohol, substances or excessive caffeine. His family history was unremarkable and did not include sudden death. There

\footnotetext{
"Corresponding author.
}

were no abnormalities on physical examination. His blood pressure was 110/65 $\mathrm{mmHg}$ and his pulse rate was 60 beats/min. His body temperature was $37.2^{\circ}$ Celsius.

The ECG revealed normal sinus rhythm of 68 beats/ min with intermediate axis, a normal PR-interval (140 $\mathrm{ms}$ ), and normal QRS-duration (80 ms) and QT-interval (360 ms), and nonspecific ST-segment abnormalities (Figure 1).

Shortly after admission, telemetric monitoring showed two short-lasting episodes of fast polymorphic ventricular tachycardia (PVT), 200 - 250 and 250 - 300 beats/min, respectively, with a short coupling interval (Figures 2(a) and (b)). The episodes of PVT were preceded and followed by sinus bradycardia (50 - 55 beats/min) and AVjunctional escape beats. However, there were neither extreme bradycardia nor prolonged QT-interval documented. The ventricular tachycardia was hemodynamically well-tolerated, but accompanied by some chest discomfort.

Cardiological workup revealed no cause for the PVT episodes: echocardiography and chest-X-ray showed no evidence of structural heart disease or cardiomyopathy, coronary angiography ruled out coronary artery disease, and laboratory examinations showed no electrolyte imbalances. Exercise testing was unremarkable, showing no exercise-induced ventricular ectopy. We were unable to establish the serum level of modafinil. The patient declined a drug challenge (ajmaline or flecainide) to investigate possible Brugada syndrome. 


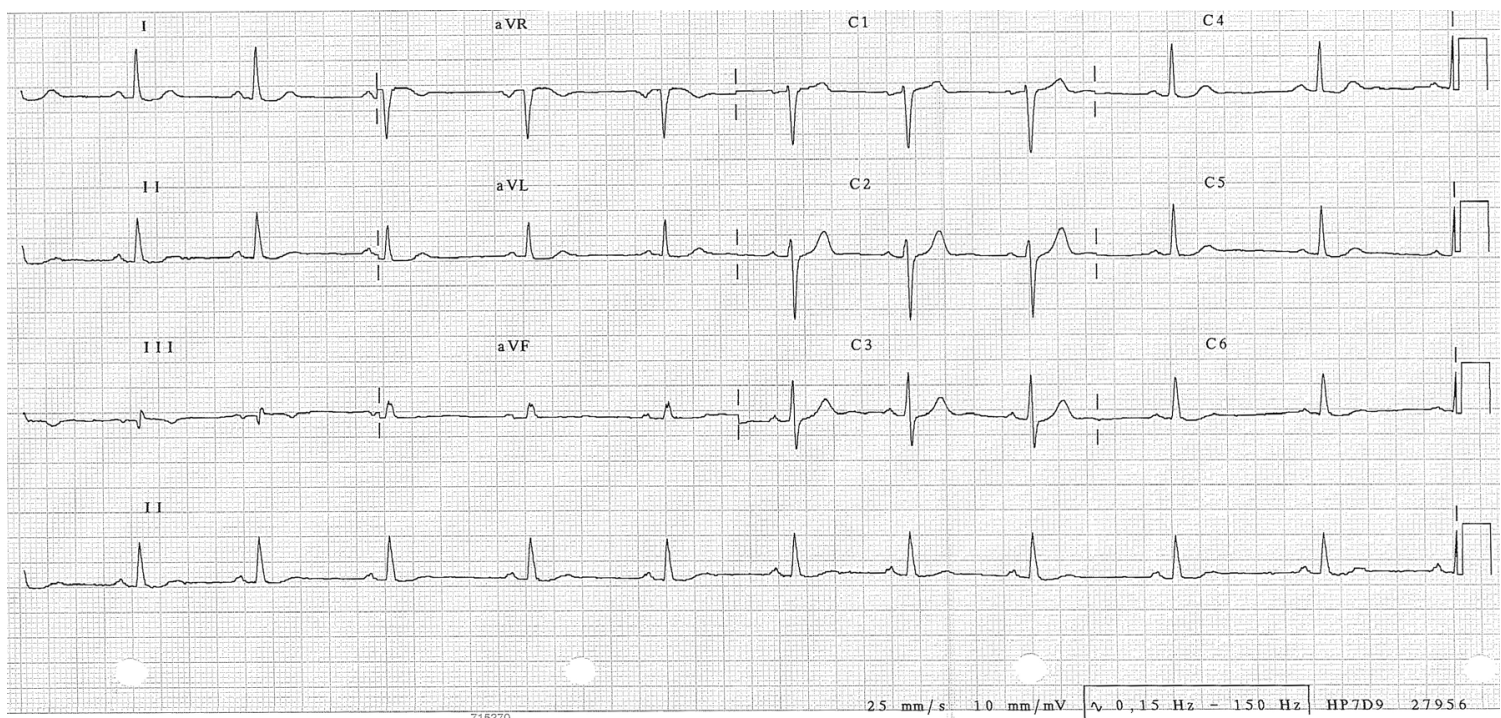

Figure 1. ECG on admission shows sinus rhythm, 68 beats/min, within normal limits.

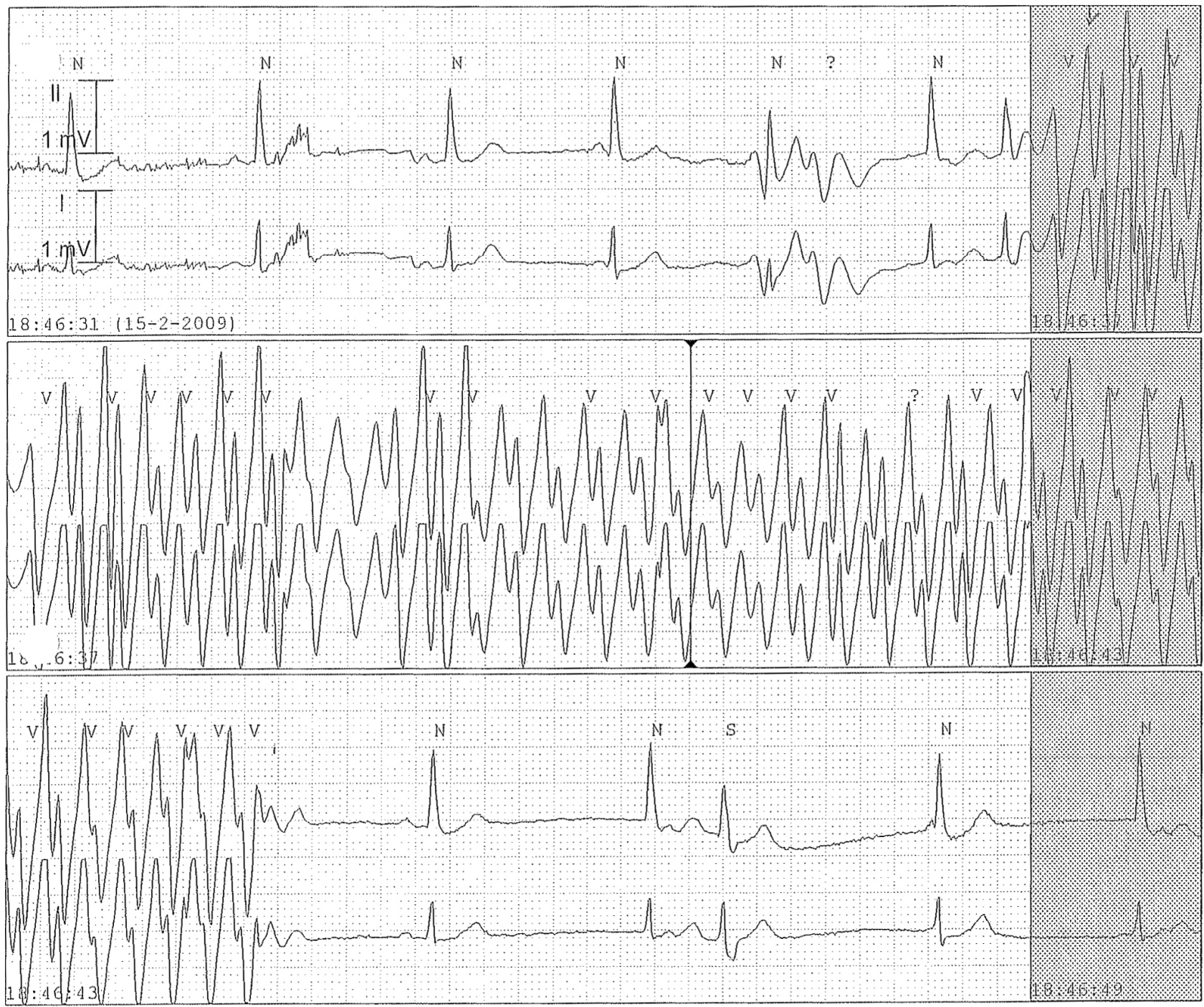




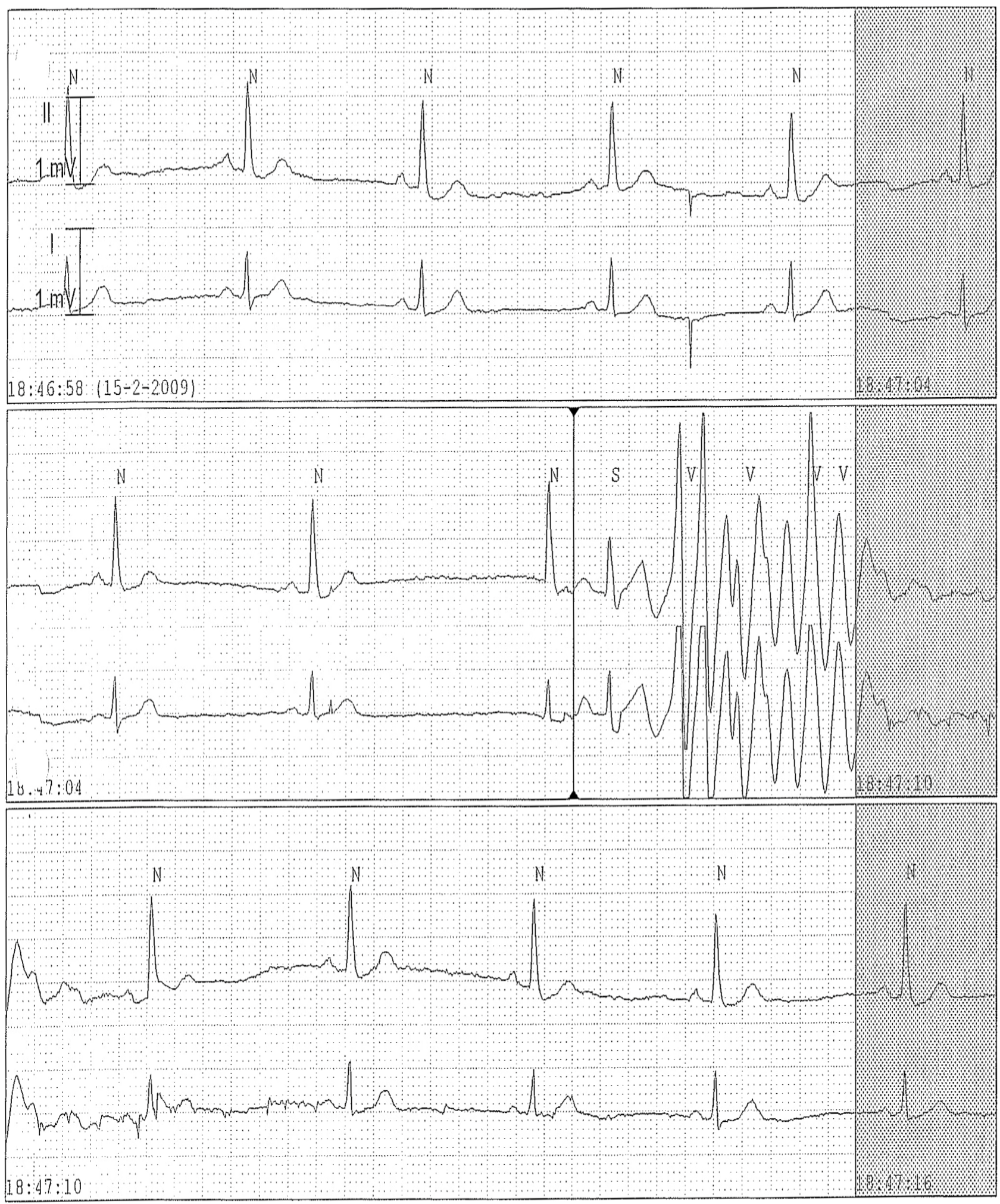

(b)

Figure 2. (a) Episode of polymorphic ventricular tachycardia (PVT), 200 - 250 beats/min, with short coupling interval. The PVT was preceded by sinus bradycardia (50 - 55 beats/min), the fourth QRS-complex is a AV-nodal escape beat. After spontaneous termination of the PVT, sinus bradycardia and AV-nodal escape beats were again documented; (b) Short episode of fast polymorphic ventricular tachycardia, 250 - 300 beats/min, with a short coupling interval. This episode was preceded by sinus bradycardia (40 - 50 beats/min) and the ninth QRS-complex is an AV-nodal escape beat. 
Having ruled out other causes of PVT, we suspected that modafinil may have contributed to their occurrence. Accordingly, we discontinued this drug. This resulted in the absence of arrhythmia reccurrence during 6 days of in-hospital monitoring. The clinical course was also otherwise uneventful, and the patient had no complaints during outpatient clinic follow-up for 6 months. He declined further investigations to rule out an inherited arrhythmia syndrome.

\section{Discussion}

We considered the observation that ventricular tachyarrhythmias did not recur after discontinuation of modafinil, while other causes for these arrhythmias were not found despite extensive cardiologic examination, evidence to suggest that these arrhythmias were caused by modafinil. Still, uncertainties remain, e.g., the question why arrhythmias were not noted before, although the patient had used this drug for some time.

Modafinil (Modiodal ${ }^{\circledR}$ ) is a recently approved, nonamphetamine-like, wake-promoting agent for narcolepsy and shift work sleep disorder and is also approved as adjunctive treatment of obstructive sleep apnea/hypopnea syndrome [1,2]. Madras and co-workers reported that modafinil occupies dopamine and norepinephrine transporters in vivo and modulates these transporters in vitro [3]. However, the exact mechanism of action is largely unknown. Modafinil is chemically and pharmacologically different from other central nervous system (CNS) stimulants $[4,6]$. It is well but slowly resorbed after oral intake, the maximal serum concentration is reached after 2 - 3 hours. This drug is metabolized predominantly by the liver into the inactive modafinil-acid and eliminated mainly by the kidneys. The halftime of elimination is 10 12 hours. The daily recommended dose is $200 \mathrm{mg}$, in case of severe liver or renal dysfunction $100 \mathrm{mg}$. Common side effects are headache, nausea, insomnia, nervousness, and, less often reported, hypertension, tachycardia, palpitations, chest pain, and liver test abnormalities. Rarely, bradycardia and ectopic beats were reported. Modafinil induces and inhibits several cytochrome P450 isoenzymes and consequently has the potential to interact with drugs from all classes [5].

In placebo-controlled clinical trials and in post-marketing surveillance, neither drug-related ECG abnormalities nor cardiac arrhythmias were documented. We found in the literature one case report on frequent premature ventricular contractions during ambulatory Holter monitoring in a 54-year-old Caucasian man who used modafinil $100 \mathrm{mg}$ twice daily for fatigue and lack of concentration, but was otherwise healthy [7]. The US-prescribing information, however, notes that caution should be exercised when prescribing modafinil to patients with a his- tory of left ventricular hypertrophy or to patients with mitral valve prolapse who have experienced the mitral valve prolapse syndrome when previously receiving CNS stimulants. Modafinil has not yet been evaluated in patients with a history of myocardial infarction, acute coronary syndromes or heart failure.

The exact causal mechanism of cardiac arrhythmias associated with modafinil remains to be elucidated. Stimulation of central dopamine receptors is considered to play an essential role, which may contribute to the induction of cardiac arrhythmias. Therefore, in general, caution is advocated on usage of recently approved CNS stimulants, like modafinil, which lack long-standing clinical evaluation, particularly with regards to conclusive information on adverse cardiovascular effects. Exploring and understanding the mechanisms of action of these drugs may enable the development of more selective compounds with fewer side effects.

\section{Conclusion}

Modafinil, a wake-promoting agent for narcolepsy, shift work sleep disorder and an adjunctive drug for treatment of obstructive sleep apnea/hypopnea syndrome, is rarely related to adverse ventricular arrhythmias, which are considered clinically important.

\section{REFERENCES}

[1] S. Nishino, "Narcolepsy: Pathophysiology and Pharmacology,” Journal of Clinical Psychiatry, Vol. 68, Suppl. 13, 2007, pp. 9-15.

[2] I. Morrison and R. L. Riha, "Excessive Daytime Sleepiness and Narcolepsy-An Approach to Investigation and Management," European Journal of Internal Medicine, Vol. 23, No. 2, 2012, pp. 110-117. doi:10.1016/j.ejim.2011.09.005

[3] B. K. Madras, Z. Xie, Z. Lin, A. Jassen, H. Panas, L. Lynch, R. Johnson, E. Livni, T. J. Spencer, A. A. Bonab, G. M. Miller and A. J. Fischman, "Modafinil Occupies Dopamine and Norepinephrine Transporters in Vivo and Modulates the Transporters and Trace Amine Activity in Vitro," Journal of Pharmacology and Experimental Therapeutics, Vol. 319, No. 2, 2006, pp. 561-569. doi:10.1124/jpet.106.106583

[4] M. J. Minzenberg and C. S. Carter, "Modafinil: A Review of Neurochemical Actions and Effects on Cognition," Neuropsychopharmacology, Vol. 33, No. 7, 2008, pp. 1477-1502. doi:10.1038/sj.npp.1301534

[5] R. Kumar, "Approved and Investigational Uses of Modafinil: An Evidence-Based Review,” Drugs, Vol. 68, No. 13, 2008, pp. 1803-1839. doi:10.2165/00003495-200868130-00003

[6] W. M. Qu, Z. L. Huang, X. H. Xu, N. Matsumoto and Y. Urade, "Dopaminergic D1 and D2 Receptors Are Essential for the Arousal Effect of Modafinil," Journal of Neuroscience, Vol. 28, No. 34, 2008, pp. 8462-8469. 
doi:10.1523/JNEUROSCI.1819-08.2008

[7] N. Oskooilar, "A Case of Premature Ventricular Contractions with Modafinil,” American Journal of Psychiatry,
Vol. 162, No. 10, 2005, pp. 1983-1984.

doi:10.1176/appi.ajp.162.10.1983-a 